\title{
Coding Prony's method in MATLAB and applying it to biomedical signal filtering
}

A. Fernández Rodríguez, L. de Santiago Rodrigo, E. López Guillén, J. M. Rodríguez Ascariz,

J. M. Miguel Jiménez and Luciano Boquete ${ }^{*}$ (i)

\begin{abstract}
Background: The response of many biomedical systems can be modelled using a linear combination of damped exponential functions. The approximation parameters, based on equally spaced samples, can be obtained using Prony's method and its variants (e.g. the matrix pencil method). This paper provides a tutorial on the main polynomial Prony and matrix pencil methods and their implementation in MATLAB and analyses how they perform with synthetic and multifocal visual-evoked potential (mfVEP) signals.

This paper briefly describes the theoretical basis of four polynomial Prony approximation methods: classic, least squares (LS), total least squares (TLS) and matrix pencil method (MPM). In each of these cases, implementation uses general MATLAB functions. The features of the various options are tested by approximating a set of synthetic mathematical functions and evaluating filtering performance in the Prony domain when applied to mfVEP signals to improve diagnosis of patients with multiple sclerosis (MS).

Results: The code implemented does not achieve 100\%-correct signal approximation and, of the methods tested, LS and MPM perform best. When filtering mfVEP records in the Prony domain, the value of the area under the receiver-operating-characteristic $(\mathrm{ROC})$ curve is 0.7055 compared with 0.6538 obtained with the usual filtering method used for this type of signal (discrete Fourier transform low-pass filter with a cut-off frequency of $35 \mathrm{~Hz}$ ).

Conclusions: This paper reviews Prony's method in relation to signal filtering and approximation, provides the MATLAB code needed to implement the classic, LS, TLS and MPM methods, and tests their performance in biomedical signal filtering and function approximation. It emphasizes the importance of improving the computational methods used to implement the various methods described above.
\end{abstract}

Keywords: Prony's method, Matrix pencil, Least squares, Total least squares, Multifocal evoked visual potentials, Multiple sclerosis

\section{Background}

\section{Prony's method}

In 1795, Gaspard de Prony [1] proposed a method to explain the expansion of gases as a linear sum of damped complex exponentials of signals that are uniformly sampled. Prony's method approximates a sequence of $N=2 \mathrm{p}$ equally spaced samples to a linear combination of $\mathrm{p}$ complex exponential functions with differing amplitudes, damping factors, frequencies and phase angles. The main contribution of this classic method is that it converts a non-linear approximation of exponential sums by solving a set of linear equations and a root-finding problem.

The conventional or polynomial Prony method consists of setting out an autoregressive model of order $\mathrm{p}$ that assumes that the value of sampled data $\mathrm{x}[\mathrm{n}]$ depends linearly on the preceding $p$ values in $\mathrm{x}$. Solving this linear system of equations obtains the coefficients of the characteristic or Prony polynomial $\phi(z)$. The roots of this polynomial yield two of the parameters of the solution (damping factors and frequency) and provide a second system of

* Correspondence: luciano.boquete@uah.es

Grupo de Ingeniería Biomédica, Departamento de Electrónica, Universidad

de Alcalá, Plaza de S. Diego, s/n, 28801 Alcalá de Henares, Spain

(c) The Author(s). 2018 Open Access This article is distributed under the terms of the Creative Commons Attribution 4.0 International License (http://creativecommons.org/licenses/by/4.0/), which permits unrestricted use, distribution, and reproduction in any medium, provided you give appropriate credit to the original author(s) and the source, provide a link to the Creative Commons license, and indicate if changes were made. The Creative Commons Public Domain Dedication waiver (http://creativecommons.org/publicdomain/zero/1.0/) applies to the data made available in this article, unless otherwise stated. 
equations to calculate the amplitude and phase of the $\mathrm{p}$ functions.

Prony's original method exactly matched the curve of p exponential terms to a dataset of $N=2 \mathrm{p}$ elements. When $N>2 \mathrm{p}$, the linear systems of equations are overdetermined and can be approximated by the least squares (LS) method [2]. The conventional least-squares method considers that in the linear system $(\mathbf{A} \cdot \mathbf{x} \approx \mathbf{b})$, only $\mathbf{b}$ (observation vector) is contaminated by noise, while $\mathbf{A}$ (coefficient matrix) is noise-free. However, generally both matrix $\mathbf{A}$ and vector $\mathbf{b}$ are noise-perturbed (in Prony's method, A and b share the same data source, see below) and, in this case, the total least-squares technique (TLS) [3] can be more advantageous.

In some cases, a problem with the Prony polynomial method is that it can be numerically unstable because of the steps that comprise the algorithm: solving an ill-conditioned matrix equation and finding the roots of a polynomial. When the number of exponentials is relatively high, the sensitivity of roots of the characteristic polynomial to perturbations of their coefficient is likewise high [4] and Prony's method may be unstable.

Another alternative is to use the matrix pencil method (MPM). Although similar to Prony's method, it consists of solving an eigenvalue problem rather than following the conventional two-step Prony method. It has been found through perturbation analysis and simulation that for signals with unknown damping factors the MPM is less sensitive to noise than the polynomial method [5].

In recent years, and due to advances in computing systems, Prony's method has been successfully applied in various engineering sectors, such as electric power quality analysis [6], materials science [7], antennae [8], etc. In the biomedical field, the classic Prony method is used in [9] to process multifocal visual-evoked potentials (mfVEPs) to diagnose the early stages of multiple sclerosis (MS). The LS Prony method is used in [10] to estimate the parameters of the single event-related potential; the TLS is used in [11] to discriminate between three cardiac problems, and the MPM is used in [12-14].

Various programming languages are widely used in the scientific field. These languages include Python, a free and open-source high-level programming language $[15,16]$, and $M_{A T L A B}{ }^{\circ}$, a proprietary product.

$\mathrm{MATLAB}^{\circ}$ is user-friendly and needs practically no formal programming knowledge [17]. As it implements a wide number and variety of functions (statistics, neural networks, graphics, wavelets, etc.), it is widely accepted as a development platform for numerical software by a significant portion of the computational science and engineering community [18-20]. Its open availability ensures reproducibility and knowledge exchange.

\section{Objectives}

This paper presents a tutorial on implementation in MATLAB of two families of Prony methods: the polynomial method (classic and extended - LS and TLS) and the matrix pencil method. It presents an overview of the mathematical bases of each method and implements them in MATLAB using the functions directly available. The results produced by the different methods when approximating synthetic signals are obtained and filtering of mfVEP records is implemented in the Prony domain. The Discussion section provides information on possible ways of mitigating the ill-conditioning problems associated with several of the resolution phases of the Prony methods.

\section{Implementation}

\section{Polynomial method}

A data sequence $x[n](n=1, \ldots \mathrm{N})$ can be represented by the sum of $p$ complex parameters (order $\mathrm{p}$ ) according to the following expression:

$$
\begin{aligned}
x[n] & =\sum_{k=1}^{p} A_{k} e^{j \theta_{k}} \cdot e^{\left(\alpha_{k}+j 2 \pi f_{k}\right) T_{s}(n-1)} \\
& =\sum_{k=1}^{p} h_{k} \cdot z_{k}^{(n-1)}
\end{aligned}
$$

Approximation of signal $x[n]$ occurs in $p$ components, in which $A_{k}$ is the initial amplitude in the same units as $x[n], \alpha_{k}$ is the damping factor in seconds $^{-1}, f_{k}$ is the frequency in Hertz, $\mathrm{T}_{\mathrm{S}}$ is the sampling period (in seconds) of signal $x[n]$ and $\theta_{k}$ is the initial phase in radians. Therefore, signal $x[n]$ is characterized by the parameters $A_{k}, \alpha_{k}, f_{k}$ and $\theta_{k}(\mathrm{k}=$ $1, \ldots, \mathrm{p}) . h_{k}$ is the time-independent component and $z_{k}$ is an exponential and time-dependent component (poles).

Equation 1 is the expression of the general solution of a homogeneous linear difference equation, if the $p$ roots are different [21]. In order to find that equation we have to construct its characteristic equation, which is

$$
\phi(z)=\prod_{k=1}^{p}\left(z-z_{k}\right)=\sum_{k=0}^{p} a[k] z^{p-k} ; \quad a[0]=1
$$


where the characteristic roots are the parameters $z_{k}$ in Eq. 1.

Demonstration of the Prony approximation method is found in [22]. Practical implementation requires performance of the following steps:

Step 1: Solve the linear prediction model constructed by the observed dataset and the obtained coefficients (a [1]...a[p]) of the characteristic polynomial. An autoregressive model of order $\mathrm{p}$ considers that the value of $\mathrm{x}[\mathrm{n}]$ depends linearly on the preceding $p$ values in $\mathrm{x}$. Equation 1 can be rewritten as a linear prediction model according to the matrix system $\mathbf{T}_{\mathrm{pxp}} \cdot \mathbf{a}_{\mathrm{px} 1}=-\mathbf{x}_{\mathrm{px} 1}$ :

$$
\begin{gathered}
\left(\begin{array}{cccc}
x[p] & x[p-1] & \cdots & x[1] \\
x[p+1] & x[p] & \cdots & x[2] \\
\vdots & \vdots & \ddots & \vdots \\
x[2 p-1] & x[2 p-2] & \cdots & x[p]
\end{array}\right)\left(\begin{array}{c}
a[1] \\
a[2] \\
\vdots \\
a[p]
\end{array}\right) \\
=-\left(\begin{array}{c}
x[p+1] \\
x[p+2] \\
\vdots \\
x[2 p]
\end{array}\right)
\end{gathered}
$$

Where.

a: Linear prediction coefficients vector.

x: Observation vector.

T: Forward linear prediction matrix (a square Toeplitz matrix).

Solving this linear system (3) reveals that the values of a are the coefficients of the characteristic or Prony polynomial $\phi(z)$.

Step 2: Find the roots of the characteristic or Prony polynomial formed from the linear prediction coefficients.

Solving an equation in finite differences is achieved by finding the roots of the characteristic polynomial. As vector $\mathbf{a}$ is known from (3), the roots $z_{k}$ of the polynomial $\phi(z)$ can be computed to obtain the damping factor $\left(\alpha_{k}\right)$ and frequency $\left(f_{k}\right)$.

$$
\begin{aligned}
\alpha_{k} & =\frac{\ln \left|z_{k}\right|}{T_{s}} \\
f_{k} & =\frac{\tan ^{-1}\left[\frac{\operatorname{Im}\left(z_{k}\right)}{\operatorname{Re}\left(z_{k}\right)}\right]}{2 \pi T_{s}}
\end{aligned}
$$

Step 3: Solve the original set of linear equations to yield the estimates of the exponential amplitude and sinusoidal phase.

First, the initial system of equations $\left(\mathbf{Z}_{\mathrm{pxp}} \cdot \mathbf{h}_{\mathrm{px} 1}=\mathbf{x}_{\mathrm{px} 1}\right)$ is solved:

$$
\left(\begin{array}{cccc}
z_{1}^{0} & z_{2}^{0} & \cdots & z_{p}^{0} \\
z_{1}^{1} & z_{2}^{1} & \cdots & z_{p}^{1} \\
\vdots & \vdots & \ddots & \vdots \\
z_{1}^{p-1} & z_{2}^{p-1} & \cdots & z_{p}^{p-1}
\end{array}\right)\left(\begin{array}{c}
h_{1} \\
h_{2} \\
\vdots \\
h_{P}
\end{array}\right)=\left(\begin{array}{c}
x[1] \\
x[2] \\
\vdots \\
x[p]
\end{array}\right)
$$

The $\mathbf{h}_{\mathbf{k}}$ values yield the amplitude $\left(A_{k}\right)$ and phase $\left(\theta_{k}\right)$ :

$$
\begin{aligned}
& A_{k}=\left|h_{k}\right| \\
& \theta_{k}=\tan ^{-1}\left[\frac{\operatorname{Im}\left(h_{k}\right)}{\operatorname{Re}\left(h_{k}\right)}\right]
\end{aligned}
$$

The classic Prony method $(\mathrm{N}=2 \mathrm{p})$ obtains an exact fit between the sampled data points and the exponentials if matrices $\mathbf{T}$ and $\mathbf{Z}$ are non-singular. However, in many practical cases $\mathrm{N}>2 \mathrm{p}$ and, in this situation, both systems are overdetermined (more equations than unknowns) and can be approximated using the LS or TLS methods.

\section{Least squares}

In general, given the overdetermined linear system: $\mathbf{A} \mathbf{x} \approx \mathbf{b}$ with $\mathbf{A} \in \mathbb{C}^{\mathrm{mxn}}, \mathbf{b} \in \mathbb{C}^{\mathrm{mx1}}, \mathbf{x} \in \mathbb{C}^{\mathrm{nx} 1}, \mathrm{~m}>\mathrm{n}$; being $\mathbf{A}$ the data matrix and $\mathbf{b}$ the observation vector, the least squares solution $\mathbf{x}_{\mathrm{LS}}$ is given by the normal equation:

$$
\mathbf{x}_{\mathrm{LS}}=\left(\mathbf{A}^{\mathrm{H}} \mathbf{A}\right)^{-1} \mathbf{A}^{\mathrm{H}} \mathbf{b}=\mathbf{A}^{+} \mathbf{b}
$$

$\mathrm{H}$ represents the Hermitian conjugate of a matrix and $\mathbf{A}^{+}$is the Moore-Penrose pseudoinverse matrix of $\mathbf{A}$. In practice, the normal equation is rarely used, as methods based on QR decomposition or singular value decomposition (SVD), among others, are preferable.

\section{Total least squares}

Solution of the system $\mathbf{A} \mathbf{x} \approx \mathbf{b}$ by the total least-squares method is a generalization of the LS approximation method when the data matrix $\mathbf{A}$ and observation vector b are contaminated with noise. In Prony's method, eqs. 3 and 6 are constructed from the measured signals. The basic total least-squares algorithm is [3]:

$\mathbf{C}:=[\mathbf{A}: \mathbf{b}]$, matrix $\mathbf{A}$ augmented (expansion by columns) by vector $\mathbf{b}\left(\mathbf{C} \in \mathbb{C}^{\mathrm{mx}(\mathrm{n}+1)}\right)$. SVD of $\mathbf{C}$ matrix is then performed:

$$
\mathbf{C}=\mathbf{U} \Sigma \mathbf{V}^{\mathbf{H}}
$$

The matrices $\mathbf{U}_{\mathrm{m} \times \mathrm{m}}$ (left singular vector matrix) and $\mathbf{V}_{(\mathrm{n}+1) \times(\mathrm{n}+1)}$ (right singular vector matrix) are orthonor$\operatorname{mal}\left(\mathbf{U}^{\mathrm{H}} \mathbf{U}=\mathbf{U U}^{\mathrm{H}}=\mathbf{I}_{m}, \quad \mathbf{V}^{\mathrm{H}} \mathbf{V}=\mathbf{V} \mathbf{V}^{\mathrm{H}}=\mathbf{I}_{n+1}\right)$ and $\boldsymbol{\Sigma}_{\mathrm{m} \times(\mathrm{n}}$ $\left.+1)=\operatorname{diag}\left(\sigma_{1}, \sigma_{2}, \ldots \sigma_{\min }\{\mathrm{m}, \quad \mathrm{n}+1\}\right)\right)$ being $\sigma_{1} \geq \sigma_{2} \ldots \geq$ $\sigma_{\min \{m, n+1\}}$ the singular values of $\mathbf{C}$.

The structure of $\mathbf{V}$ is as follows: 


$$
\mathbf{V}=\left[\begin{array}{ccc}
\mathrm{v}_{1.1} & \cdots & \mathrm{v}_{1,(\mathrm{n}+1)} \\
\vdots & \ddots & \vdots \\
\mathrm{v}_{(\mathrm{n}+1), 1} & \cdots & \mathrm{v}_{(\mathrm{n}+1),(\mathrm{n}+1)}
\end{array}\right]
$$

The TLS solution exists if $v_{(n+1),}(n+1) \neq 0$ [23] and, moreover it is unique if $\sigma_{n} \neq \sigma_{n+1}$ :

$$
\mathbf{x}_{\mathrm{TLS}}=-\frac{1}{\mathrm{v}_{(\mathrm{n}+1),(\mathrm{n}+1)}}\left[\begin{array}{llll}
\mathrm{v}_{1,(\mathrm{n}+1)}, v_{2,(n+1)} & \cdots & \mathrm{v}_{\mathrm{n},(\mathrm{n}+1)}
\end{array}\right]^{\mathrm{T}}
$$

Algorithms in which the solution does not exist or is not unique are considered in detail in [24].

\section{Implementation in MATLAB of the polynomial method}

The code presented was developed and tested under MATLAB R2016b. Code 1 presents implementation in MATLAB of a function to perform the Prony approximation using the three polynomial methods mentioned above. The function is defined as follows:

\section{function [Amp,alpha,freq,theta $]=$ polynomial_-} method (x,p,Ts,method)

The sampled data are given in vector $\mathrm{x} ; p$ is the number of terms to obtain in the approximation, Ts is the sampling time of the signal and classic, LS or TLS indicates the method used to solve the problem. The function returns the parameters (Amp, alpha, freq, theta) resulting from the approximation.

First, the sample length is obtained $(\mathbf{N}=$ length $(\mathbf{x}))$ and consistency between the parameter method, $p$ and the sample data length is checked.

\section{Step 1.}

Coding the linear system of Eq. 2 takes into account that the MATLAB function $\boldsymbol{T}=\operatorname{toeplitz}(c, r)$ creates non-symmetrical Toeplitz matrix $\mathbf{T}$ (dimensions $p \times p$ under the classic method and $(N-p) \times p$ under the overdetermined methods), having $c$ as its first column and $r$ as its first row, achieved by the following instruction:

$\mathrm{T}=$ toeplitz $(\mathbf{x}(\mathbf{p}: \mathrm{N}-1), \mathbf{x}(\mathbf{p}:-1: 1))$;

The solution of this system of eqs. (T.a $=-\mathbf{x})$ for the classic and LS methods is obtained in MATLAB using the matrix left division (also known as backslash) operator. If $\mathbf{T}$ is square and if it is invertible, the backslash operator solves the linear equations using the $Q R$ method. With an overdetermined system, LS should be used. The backslash operator is a collection of algorithms used to solve a linear system [25], selected according to the characteristics of matrix $\mathbf{T}$. Taking into account that vector $\mathbf{x}$ is a matrix column:

$\mathbf{a}=-\mathbf{T} \backslash \mathbf{x}(\mathbf{p}+\mathbf{1 : N})$;

In the case of the TLS option, the function $\mathbf{a}=$ $\operatorname{tls}(T,-x(p+1: N))$; is called (Code 2$)$.

Step 2.

The $\mathrm{p}$ roots of the polynomial are now obtained:

$$
z^{p}+a[1] z^{p-1}+\ldots+a[p]=0
$$

The MATLAB instruction $r=\operatorname{roots}(c)$ returns a column vector whose elements are the roots of the polynomial c. Row vector c contains the coefficients of a polynomial, ordered in descending powers. If $c$ has $n+1$ components, the polynomial it represents is $c_{1} s^{n}+\ldots+$ $\mathrm{c}_{\mathrm{n}} \mathrm{S}+\mathrm{c}_{\mathrm{n}+1}$.

The input vector for the roots function must be a row vector and must contain the element $a[0]=1$, which was not obtained in the previous solution. Its implementation is therefore.

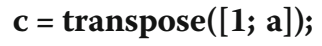

Based on $r$, and having defined the acquisition period Ts, it is possible to find the values of the damping factor $\left(\alpha_{k}\right)$ and frequency $\left(f_{k}\right)$ :

alpha $=\log (\operatorname{abs}(\mathbf{r})) /$ Ts;

freq $=\operatorname{atan} 2(\operatorname{imag}(r), \operatorname{real}(\mathbf{r})) /\left(2 * \mathrm{pi}^{*} \mathrm{Ts}\right)$;

$\log$ is the Napierian logarithm and atan 2 returns the four-quadrant inverse tangent.

Step 3: Obtain complex parameters $h_{k}$ from roots $z_{k^{*}}$ The number of equations (len_vandermonde) employed for the solution is set ( $\mathrm{p}$ in classic and $\mathrm{N}$ in overdetermined systems) and the data matrix for the system of equations is constructed (6):

$\mathrm{Z}=\operatorname{zeros}($ len_vandermonde,p);

for $i=1$ :length(r).

$\mathrm{Z}(:, \mathbf{i})=\operatorname{transpose}\left(\mathbf{r}(\mathbf{i}){ }^{\wedge}(0:\right.$ len_vandermonde-1));

End

Finally, the following is solved:

$\mathbf{h}=\mathbf{Z} \backslash \mathbf{x}(1$ :len_vandermonde);

In the case of the TLS option, the function $\mathbf{h}=\mathbf{t} \mathbf{l} \mathbf{s}(\mathbf{Z}$, $\mathbf{x}(\mathbf{1}$ : len_vandermonde)); (Code 2 ) is called. In the TLS algorithm, SVD is used. The infinite values therefore have to be converted into maximum representative values beforehand otherwise the SVD function will yield an error.

The solutions yield the initial amplitude $\left(A_{k}\right)$ and initial phase $\left(\theta_{k}\right)$ values:

$$
\operatorname{Amp}=\operatorname{abs}(\mathbf{h}) \text {; }
$$$$
\text { theta }=\operatorname{atan} 2(\mathrm{imag}(\mathrm{h}), \operatorname{real}(\mathbf{h})) \text {; }
$$

The function that solves a linear system using the TLS method (Code 2) receives as arguments matrices $\mathbf{A}$ and b, which define the linear system to solve: Function $\mathbf{x}=$ $\operatorname{tls}(\mathbf{A}, \mathbf{b})$. The number of columns in matrix $\mathbf{A}$ is obtained $([\sim, \mathbf{n}]=\operatorname{size}(\mathbf{A}) ;)$ and augmented matrix $\mathbf{C}(\mathbf{C}$ $\left.=\left[\begin{array}{ll}\mathbf{A} & \mathbf{b}\end{array}\right]\right)$ is constructed while matrix $\mathbf{V}$ of the SVD decomposition is obtained via the instruction $[\sim, \sim, V]=$ $\operatorname{svd}(\mathbf{C})$; the TLS solution (if it exists) is obtained by applying the formula (12) to matrix $\mathbf{V}$. 

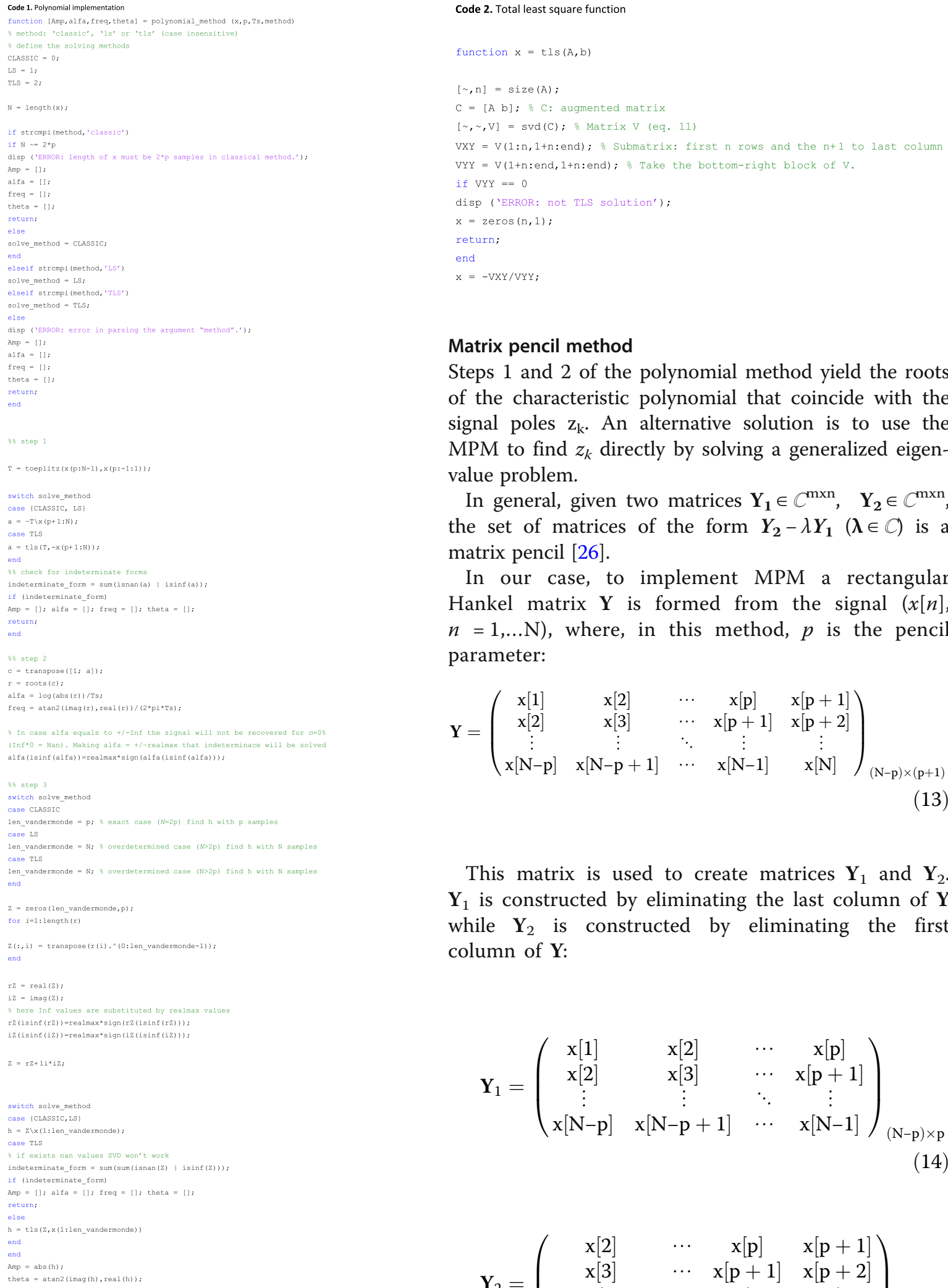

\section{Matrix pencil method}

Steps 1 and 2 of the polynomial method yield the roots of the characteristic polynomial that coincide with the signal poles $z_{k}$. An alternative solution is to use the MPM to find $z_{k}$ directly by solving a generalized eigenvalue problem.

In general, given two matrices $\mathbf{Y}_{1} \in \mathbb{C}^{\mathrm{mxn}}, \mathbf{Y}_{\mathbf{2}} \in \mathbb{C}^{\mathrm{mxn}}$, the set of matrices of the form $Y_{2}-\lambda Y_{1}(\lambda \in \mathbb{C})$ is a matrix pencil [26].

In our case, to implement MPM a rectangular Hankel matrix $\mathbf{Y}$ is formed from the signal $(x[n]$, $n=1, \ldots \mathrm{N})$, where, in this method, $p$ is the pencil parameter:

$\mathbf{Y}=\left(\begin{array}{ccccc}\mathrm{x}[1] & \mathrm{x}[2] & \cdots & \mathrm{x}[\mathrm{p}] & \mathrm{x}[\mathrm{p}+1] \\ \mathrm{x}[2] & \mathrm{x}[3] & \cdots & \mathrm{x}[\mathrm{p}+1] & \mathrm{x}[\mathrm{p}+2] \\ \vdots & \vdots & \ddots & \vdots & \vdots \\ \mathrm{x}[\mathrm{N}-\mathrm{p}] & \mathrm{x}[\mathrm{N}-\mathrm{p}+1] & \cdots & \mathrm{x}[\mathrm{N}-1] & \mathrm{x}[\mathrm{N}]\end{array}\right)_{(\mathrm{N}-\mathrm{p}) \times(\mathrm{p}+1)}$

This matrix is used to create matrices $\mathbf{Y}_{1}$ and $\mathbf{Y}_{2}$. $\mathbf{Y}_{1}$ is constructed by eliminating the last column of $\mathbf{Y}$ while $\mathbf{Y}_{2}$ is constructed by eliminating the first column of $\mathbf{Y}$ :

$$
\begin{aligned}
& \mathbf{Y}_{1}=\left(\begin{array}{cccc}
\mathrm{x}[1] & \mathrm{x}[2] & \cdots & \mathrm{x}[\mathrm{p}] \\
\mathrm{x}[2] & \mathrm{x}[3] & \cdots & \mathrm{x}[\mathrm{p}+1] \\
\vdots & \vdots & \ddots & \vdots \\
\mathrm{x}[\mathrm{N}-\mathrm{p}] & \mathrm{x}[\mathrm{N}-\mathrm{p}+1] & \cdots & \mathrm{x}[\mathrm{N}-1]
\end{array}\right) \begin{array}{r}
(\mathrm{N}-\mathrm{p}) \times \mathrm{p} \\
(14)
\end{array} \\
& \mathbf{Y}_{2}=\left(\begin{array}{cccc}
\mathrm{x}[2] & \cdots & \mathrm{x}[\mathrm{p}] & \mathrm{x}[\mathrm{p}+1] \\
\mathrm{x}[3] & \cdots & \mathrm{x}[\mathrm{p}+1] & \mathrm{x}[\mathrm{p}+2] \\
\vdots & \ddots & \vdots & \vdots \\
\mathrm{x}[\mathrm{N}-\mathrm{p}+1] & \cdots & \mathrm{x}[\mathrm{N}-1] & \mathrm{x}[\mathrm{N}]
\end{array}\right)_{(\mathrm{N}-\mathrm{p}) \times \mathrm{p}}
\end{aligned}
$$


obtained from the normalized root-mean-square error is used:

$$
G=1-\frac{\left\|g_{i}[n]-\widetilde{g_{i}[n]}\right\|}{\left\|g_{i}[n]-\overline{g_{i}}\right\|}
$$

\|.\|l indicates the 2-norm and $\bar{g}_{i}$ is the mean of the reference signal.

If for a certain function $G \geq 0.60$ is fulfilled, the approximation is considered correct. Table 1 shows the number of functions correctly approximated by the Prony LS, Prony TLS and MPM methods and for the two different parameters $(\mathrm{N}, \mathrm{p})$.

None of the methods implemented works $100 \%$ correctly $\left(G \geq 0.60\right.$ for the $1000 g_{i}[n]$ functions in all the situations tested). If the mean number of functions well-approximated by each method is considered, the best result is obtained with MPM $(\overline{M P M}=999.55)$ and the worst is obtained with TLS $(\overline{T L S}=677.39)$. The LS method yields the correct approximation in $60.52 \%$ of cases, the TLS method in $2.63 \%$ of cases and the MPM method in $92.10 \%$ of cases tested in this experiment.

In general, the results obtained using LS and MPM are very similar, as the MATLAB $\operatorname{roots}(\cdot)$ function generates the companion matrix of the polynomial and uses the QR-algorithm to obtain its eigenvalues.

Figure 1 shows the roots obtained using the LS and MPM methods for one of the $g_{i}[n]$ signals $(N=$ $256, p=30$ ). The correct number of roots for signal $g_{i}[n]$ is $M=19$; in both examples, $p=30$ roots are obtained, though with the MPM method 12 roots are equal to 0 . This is because in the LS method the range of the companion matrix is always equal to $p$ and, consequently, $p$ roots are obtained. In the MPM method, the range of matrix $\left(\mathbf{Y}_{1}^{+} \mathbf{Y}_{2}\right)$ is less than or equal to $\mathrm{p}\left(\mathrm{r}=\operatorname{rank}\left(\mathbf{Y}_{1}^{+} \mathbf{Y}_{2}\right) \leq p\right)$ and $\mathrm{r}$ roots other than zero and (p-r) roots equal to 0 are obtained [5]. In the example shown, $r=18$ is fulfilled. The differences in the results between the two methods are evident in Step 3 and are due to computational errors.

\section{mfVEP filtering}

The mfVEP technique [28] can be used to obtain the electrophysiological response of the primary visual cortex to stimuli produced in a large number (e.g. 60) of sectors of the visual field. Generation of the visual stimulus is governed by the same pseudorandom sequence [29] used to
Table 1 Result of approximation of synthetic functions

\begin{tabular}{|c|c|c|c|c|}
\hline \multicolumn{5}{|c|}{ Number of functions $g_{i}[n]$ correctly approximated } \\
\hline $\mathrm{N}$ & $\mathrm{p}$ & LS & TLS & MPM \\
\hline \multirow[t]{10}{*}{1024} & 30 & 902 & 811 & 990 \\
\hline & 40 & 868 & 499 & 1000 \\
\hline & 50 & 826 & 499 & 1000 \\
\hline & 100 & 997 & 322 & 1000 \\
\hline & 150 & 1000 & 315 & 1000 \\
\hline & 200 & 1000 & 375 & 1000 \\
\hline & 250 & 1000 & 358 & 1000 \\
\hline & 300 & 1000 & 288 & 1000 \\
\hline & 400 & 1000 & 224 & 1000 \\
\hline & 500 & 999 & 137 & 1000 \\
\hline \multirow[t]{10}{*}{512} & 30 & 941 & 741 & 1000 \\
\hline & 40 & 974 & 660 & 1000 \\
\hline & 50 & 996 & 682 & 1000 \\
\hline & 60 & 999 & 618 & 1000 \\
\hline & 70 & 1000 & 544 & 1000 \\
\hline & 100 & 1000 & 565 & 1000 \\
\hline & 150 & 1000 & 622 & 1000 \\
\hline & 200 & 1000 & 579 & 1000 \\
\hline & 220 & 1000 & 517 & 1000 \\
\hline & 250 & 999 & 516 & 1000 \\
\hline \multirow[t]{10}{*}{256} & 30 & 984 & 909 & 1000 \\
\hline & 40 & 998 & 872 & 1000 \\
\hline & 50 & 998 & 855 & 1000 \\
\hline & 60 & 1000 & 826 & 1000 \\
\hline & 70 & 1000 & 778 & 1000 \\
\hline & 80 & 1000 & 862 & 1000 \\
\hline & 90 & 1000 & 827 & 1000 \\
\hline & 100 & 1000 & 758 & 1000 \\
\hline & 110 & 1000 & 733 & 1000 \\
\hline & 120 & 996 & 758 & 1000 \\
\hline \multirow[t]{5}{*}{128} & 20 & 994 & 995 & 994 \\
\hline & 30 & 1000 & 960 & 1000 \\
\hline & 40 & 1000 & 956 & 1000 \\
\hline & 50 & 1000 & 931 & 1000 \\
\hline & 60 & 1000 & 910 & 1000 \\
\hline \multirow[t]{3}{*}{64} & 20 & 1000 & 1000 & 999 \\
\hline & 25 & 1000 & 969 & 1000 \\
\hline & 30 & 1000 & 970 & 1000 \\
\hline \multicolumn{2}{|c|}{$\begin{array}{l}\text { Average value per } \\
\text { method }\end{array}$} & $\overline{\mathrm{LS}}=986.08$ & $\overline{\mathrm{TLS}}=677.39$ & $\overline{\mathrm{MPM}}=999.55$ \\
\hline
\end{tabular}



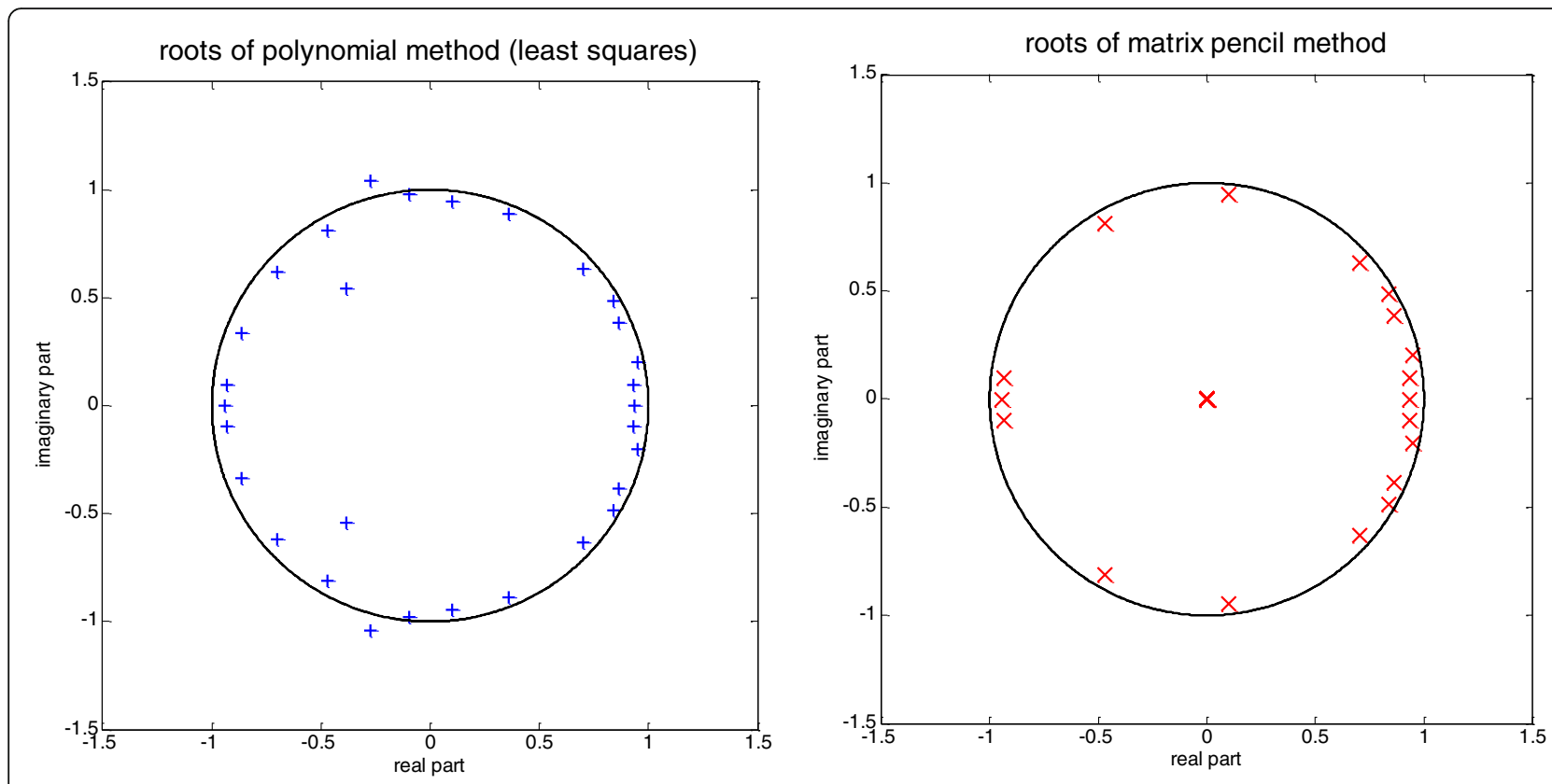

Fig. 1 Poles obtained using the polynomial (LS) and MPM methods

separate the individual responses of each sector from the continual EEG recording obtained using electrodes. Analysis of mfVEP signals is employed in diagnosis and study of patients with glaucoma, amblyopia, nerve optic drusses, optic neuritis, multiple sclerosis and other pathologies.

The aim of this test is to evaluate whether mfVEP signal filtering in the Prony domain improves the separation between the signals of control subjects and the signals of patients with MS. It uses the signal-to-noise ratio (SNR) of the records as the parameter. The discrimination factor is evaluated using the area under the ROC curve (AUC). The results achieved by applying the conventional method to mfVEP records are then compared: signals filtered using the discrete Fourier transform (DFT) between 0 and $35 \mathrm{~Hz}$ and the signals filtered in the Prony domain.

This experiment uses a database of mfVEP signals captured from 28 patients (age $34.39 \pm 10.09$ years, 7 males and 21 females) diagnosed with MS according to the McDonald criteria; the signals were obtained from 44 eyes in 22 control subjects (age $30.20 \pm 7.55$ years, 10 males and 12 females) with normal ophthalmologic and neurological examination results. The study protocol adhered to the tenets of the Declaration of Helsinki and was approved by the local Institutional Review Board (Comité de Ética en Investigación Clínica del Hospital Universitario
Príncipe de Asturias, Alcalá de Henares, Spain). Written informed consent was obtained from all participants.

mfVEP signals were recorded monocularly with VERIS software 5.9 (Electro-Diagnostic Imaging, Inc., Redwood City, CA). The visual stimulus was a scaled dartboard with a diameter of 44.5 degrees, containing 60 sectors, each with 16 alternating checks. The luminance for the white and black checks were 200 and < $3 \mathrm{~cd} / \mathrm{m}^{2}$, respectively. The checks in each sector were reversed in contrast using a pseudorandom sequence (m-sequence) at a frame rate of $75 \mathrm{~Hz}$.

The mfVEP signals were obtained using gold cup electrodes (impedance $<2 \mathrm{~K} \Omega$ ). The reference electrode was positioned on the inion $\left(E_{R}\right)$ and the ground electrode on the forehead. The active electrodes were placed $4 \mathrm{~cm}$ above the inion $\left(\mathrm{E}_{\mathrm{A}}\right)$ and 1 $\mathrm{cm}$ above and $4 \mathrm{~cm}$ either side of the inion $\left(E_{B}\right.$, $\left.E_{C}\right)$. The difference between the signals of the active electrodes was used to obtain channels $\mathrm{CH}_{1}=$ $\mathrm{E}_{\mathrm{A}}-\mathrm{E}_{\mathrm{R}}, \mathrm{CH}_{2}=\mathrm{E}_{\mathrm{B}}-\mathrm{E}_{\mathrm{R}}$ and $\mathrm{CH}_{3}=\mathrm{E}_{\mathrm{C}}-\mathrm{E}_{\mathrm{R}}$. Three additional derived channels were obtained $\left(\mathrm{CH}_{4}=\right.$ $\left.\mathrm{CH}_{1}-\mathrm{CH}_{2}, \quad \mathrm{CH}_{5}=\mathrm{CH}_{1}-\mathrm{CH}_{3}, \quad \mathrm{CH}_{6}=\mathrm{CH}_{2}-\mathrm{CH}_{3}\right)$. Therefore, the data from 6 channels were processed. In the analogue phase, the signals were amplified at a gain of $10^{5}$ at a bandwidth between 3 and $100 \mathrm{~Hz}$. The sampling frequency was $1200 \mathrm{~Hz}$, obtaining 600 samples in each recording (length $500 \mathrm{~ms})$. 


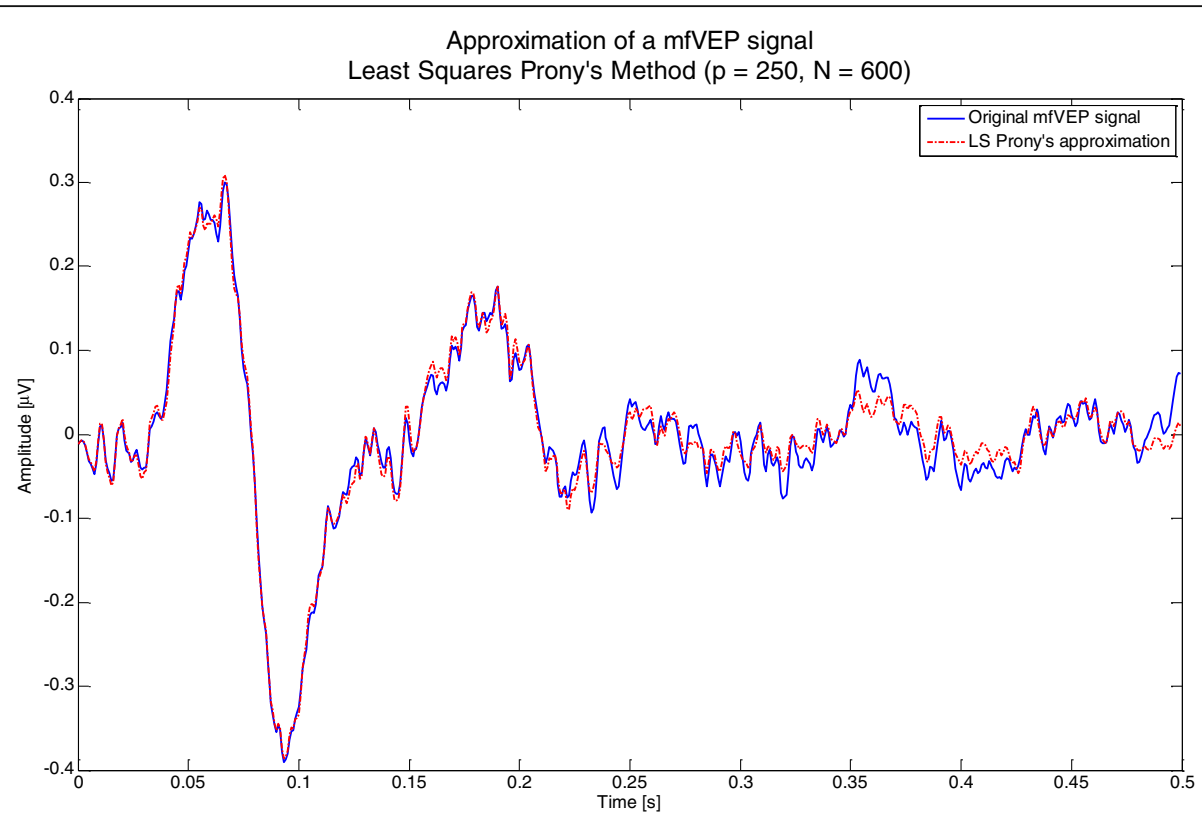

Fig. 2 Example of approximation of an mfVEP signal using Prony's method (LS)

\section{Discussion}

In this paper we have used general MATLAB functions to implement the principal methods of function approximation based on the linear combination of exponentials: the polynomial Prony method (classic, LS and TLS) and the matrix pencil method. In the polynomial method, signal poles (frequencies and damping factors) are found as roots of a polynomial while the MPM obtains the poles by finding the eigenvalues of a matrix pencil.
Currently, the most common method is Fourier analysis, which represents a signal as a summation of continuous undamped sinusoidal functions with frequency and integer times the fundamental frequency (harmonics). In contrast, the $\mathrm{p}$ components of a Prony series may be complex exponentials. In general, the Prony spectrum will be non-uniformly spaced in the frequency scale (as it is one of the estimated parameters), depending on the observed data [30].

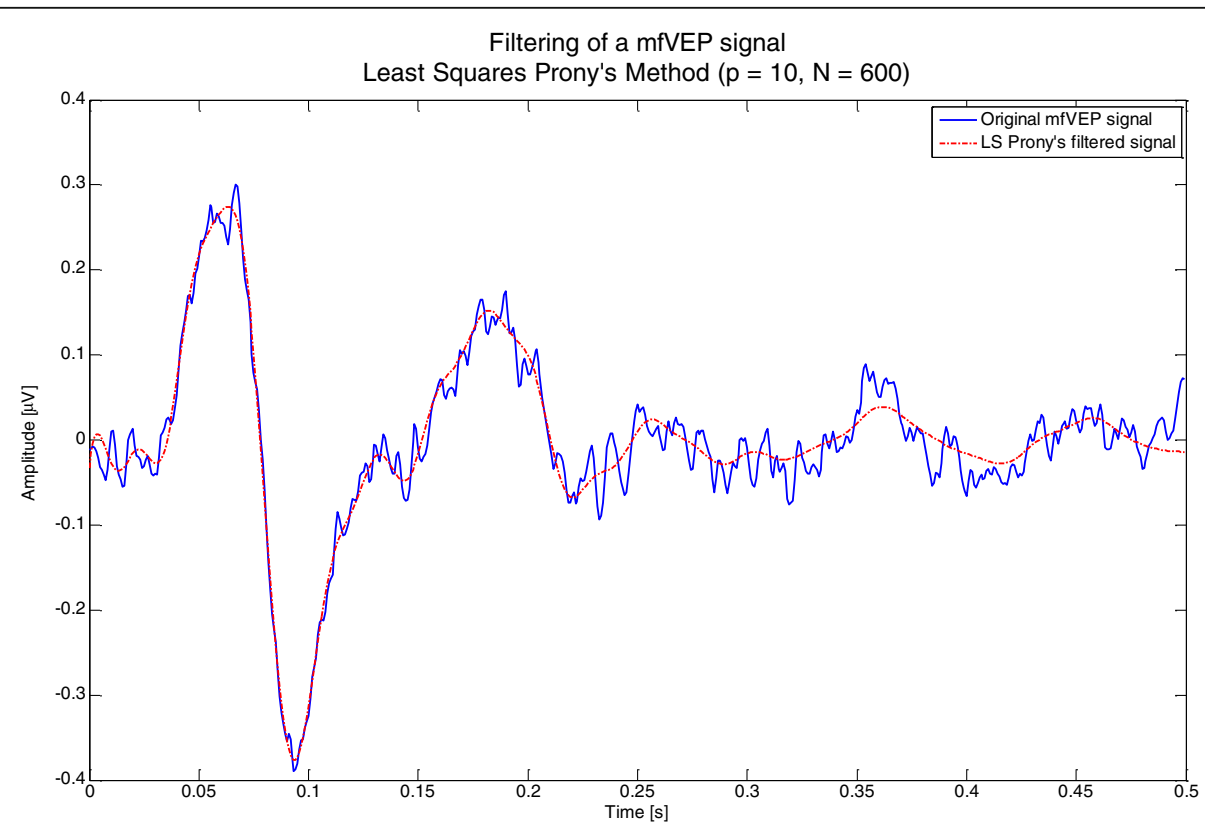

Fig. 3 Example of Prony filtering of an mfVEP signal 
Table 2 Results of filtering mfVEP signals ( $N=600, p=250, T_{s}=1 / 1200 s$ )

\begin{tabular}{lllrrr}
\hline & Well-approximated control signals (\%) & \multicolumn{2}{l}{$\begin{array}{l}\text { Well-approximated MS signals } \\
(\%)\end{array}$} & SNR $_{\text {CONTROLS }}$ & SNR \\
\hline DFT & - & - & $3.59 \pm 2.89$ & $2.44 \pm 2.11$ & 0.6538 \\
LS & $99.57 \%$ & $99.91 \%$ & $4.95 \pm 4.19$ & $2.85 \pm 2.49$ & 0.7055 \\
TLS & $48.79 \%$ & $42.90 \%$ & $3.65 \pm 2.89$ & $2.54 \pm 2.24$ & 0.6472 \\
MPM & $99.57 \%$ & $99.91 \%$ & $4.95 \pm 4.19$ & $2.85 \pm 2.49$ & 0.7055 \\
\hline
\end{tabular}

Prony modelling produces higher frequency resolution than DFT methods due to its reliance on autoregressive modelling [31]. Another advantage is that it is a natural transformation for impulse responses since it uses damped sinusoids as a basis and therefore representation is efficient in terms of the number of coefficients required [32].

Not all mathematical signals can be approximated using Prony's method [33] and computational finite arithmetic also generates errors. Consequently, the results of computational implementation of the Prony methods depend on the characteristics and number of points of the signal to be interpolated, on the $\mathrm{p}$ number of functions and on the use of computational procedures not susceptible to ill-conditioning issues. Furthermore, these potentially ill-conditioned operations are concatenated, thereby increasing the instability issues. For example, since the second step of Prony's method is an ill-conditioned problem and round-off errors must exist for the linear prediction parameters to be computed in the first step, the estimation of $z_{k}$ in the second step of Prony's method can contain significant error [34].

In our experimental approximation of synthetic functions, the best result was obtained using the MPM and LS methods, while the effectiveness of the TLS method was shown to be highly dependent on the number of points and on the $\mathrm{p}$ number of functions (Table 1). In some cases, when the number of exponentials is relatively high, the sensitivity of roots of the characteristic polynomial to perturbations of their coefficient is likewise high [4] and Prony's method may be unstable.

In a second experiment, we low-pass-filtered mfVEP signals in the Prony domain in order to evaluate the improvement in the capacity to discriminate between signals of control subjects and those of MS patients. Selecting the first 10 components of each record reveals that the AUC value between the signals of healthy subjects and those of MS subjects increases by between $0.3 \%$ and $4.7 \%$ depending on the method compared. The smallest improvement was obtained with the TLS method and the greatest improvement with the LS and MPM methods.

Coding in MATLAB used the functions directly available in this programming language. However, these evidently have their computational limitations and could be replaced with better alternatives. Various aspects that could improve the code presented in this paper are discussed below.

\section{Solution of linear systems}

Solution of the linear systems using the classic and LS methods was implemented with the MATLAB mldivide ( $)$ operator. Although the mldivide operator is valid for most cases (it selects between the LU, Cholesky, LDLT or QR-factorization methods, among others, depending on the characteristics of matrix A [35]), it may be more efficient to implement other algorithms.

The numerical stability of the solution in linear algebra may be evaluated by the condition number and the numerical rank of matrix $\mathbf{A}$. The condition number is defined as: $k_{2}(\mathbf{A})=\frac{\sigma_{\max }}{\sigma_{\min }}$; a low condition number usually means that the system is well-conditioned. The rank (r) of a matrix is the number of linear independent rows (or columns) (r $\leq \min \{\mathrm{m}, \mathrm{n}\})$ and is equal to the number of singular values $\left(\sigma_{i}\right)$ in the matrix other than zero. When $\mathrm{r}=\min \{\mathrm{m}, \mathrm{n}\}$ the matrix has full range, otherwise it is rank-deficient. If $\mathbf{A}$ is nearly rank-deficient $\left(\sigma_{\min }\right.$ is small), then the solution $\mathbf{x}$ is ill-conditioned and possibly very large. A more robust solution to obtain the effective rank may be to evaluate the number of singular values of $\mathbf{A A}^{\mathbf{H}}$ or $\mathbf{A}^{\mathbf{H}} \mathbf{A}$ above a specified tolerance. Analysing the condition number and the rank of a matrix may make it possible to select the best method for system solution.

\section{Least squares}

In general, although the normal equation is the fastest method it is not used to solve systems by LS as it yields worse numerical results than other methods. In the normal equation, accuracy depends on $k_{2}\left(\mathbf{A A}^{\mathbf{H}}\right)=k_{2}^{2}(\mathbf{A})$, although this method may be used if $\mathbf{A}$ is well-conditioned [36]. If $\mathbf{A}$ is rank-deficient, then $\mathbf{x}=\mathbf{A} \backslash \mathbf{B}$ is not necessarily the minimum norm solution. The more computationally expensive $\boldsymbol{x}=\operatorname{pinv}(\boldsymbol{A}) * \boldsymbol{B}$ computes the minimum norm least-squares solution. Specifically, the function $\operatorname{pinv}(\boldsymbol{A}$, tol) returns the Moore-Penrose pseudoinverse, obtained by SVD decomposition where the values above tolerance (tol) are set to zero; this may be adapted to an ill-conditioned problem ( $\mathbf{A}$ is not of full rank). Another 
option to obtain the Moore-Penrose pseudoinverse is proposed in [37], which makes use of QR-factorization and an algorithm based on a reverse order law for generalized inverse matrices; this method was later refined in [38]. An iterative solution to obtain the Moore-Penrose pseudoinverse was published in [39].

\section{TLS}

The TLS method implemented is the one that yielded the worst computational performance. This method performs SVD of the augmented matrix $\mathbf{C}:=[\mathbf{A}: \mathbf{b}]$; If $\mathbf{C}$ is rank-deficient or nearly rank-deficient (its singular values decay gradually), it may be advisable to truncate its small singular values [40]. [41] presents basic information, references and applications for the TLS method. In [42], an interactive method is proposed which combines orthogonal projections to a sequence of generalized Krylov subspaces of increasing dimensions and Newton's method. The introduction to [43] presents various alternatives to obtaining the solution using the TLS method and the authors present a solution based on randomized algorithms.

\section{Roots}

Numerical solution of a polynomial is a classic problem in mathematical research [44]. Methods available with which to obtain the roots of a polynomial include Laguerre [45], Bairstow, Græffe and Müller, Horner, Jenkins and Traub, and Newton [46], etc., with differing performances in terms of accuracy, convergence and speed. The code presented uses the roots() function used by the QR-algorithm on the balanced Frobenius companion matrix to compute its eigenvalues.

\section{Eigenvalues and SVD}

The eigenvalues of a square matrix $\mathbf{A}$ are the roots of its characteristic polynomial $\operatorname{det}(\mathbf{A}-\lambda \mathbf{I})=0$. However, singular values of $\mathbf{A}$ are non-negative square roots of eigenvalues of $\left(\mathbf{A}^{\mathrm{T}} \mathbf{A}\right)$, meaning that both methods are related. The general idea is to diagonalize the target matrix as the values of the diagonal are the eigenvalues. All methods to solve the eigenvalue problem are of an iterative nature [47]. The built-in MATLAB function eig(A) uses the generalized Schur decomposition method (implemented via the QR-algorithm or its variants), which consists of interactively obtaining an upper triangular matrix $\mathbf{U}$, in which the values of its diagonal are the eigenvalues of $\mathbf{A}$. The $\mathrm{QR}$-algorithm can be adapted to small or moderately large non-symmetrical eigenvalue problems [48]. For large matrices, [49] provides possible alternatives.

\section{Prony-like methods}

Other modifications have been made to the Prony method, generally with the intention of improving its numerical stability. If any of the parameters of equation (1) are known, the Prony method makes it easier to find a robust solution. In the polynomial method, small variations in the coefficients of equation (2) due to signal noise can result in large variations in its zeros and, consequently, the frequencies of the approximation will vary greatly. Parametric spectral estimation techniques, such as MUSIC (MUltiple SIgnal Classification) [50], ESPRIT (Estimation of Signal Parameters via Rotational Invariance Techniques) [51] or fast ESPRIT [52] offer an alternative that in many cases make it possible to obtain more robust solutions. [53] presents an algorithm for the factorization of a matrix pencil based on QR decomposition of a rectangular Hankel matrix, which simplifies the ESPRIT method.

The NAFASS (Non-orthogonal Amplitude Frequency Analysis of the Smoothed Signals) approach [54] makes it possible to obtain the set of frequencies that make up strongly correlated random sequences with $N<1500$. [55] presents the physical interpretation of the Prony decomposition as a linear recording of memory effects that can exist in random sequences in which the Fourier decomposition is a partial case. [56] improves the NAFASS method, presenting a linear recurrence expression that obtains the set of frequencies.

Another way to obtain more robust results is to act on the signals before obtaining their decomposition in a Prony series by using pre-filtering [57]. In the modified instantaneous Prony method [58] the input data used in an application of speech parameter extraction are those derived from the signal $x[n]$ instead of adjacent samples.

Applying the Prony method to a time window that can be moved along the $\mathrm{x}[\mathrm{n}]$ signal makes it possible to perform time-frequency analysis. One such example could be the short-time matrix pencil method (STMPS) successfully used to obtain antennae responses [59]. The Piecewise Prony Method [60] essentially consists of dividing the signal to be interpolated into windows of variable length and sampling rate.

\section{Conclusions}

Decomposition of a signal using Prony's method can be considered a generalization of the Fourier decomposition. Although the method has been known since 1795 , its application in engineering has only increased since about the 1970s as computer use has grown. This paper has presented the theoretical bases and their piece-by-piece implementation in MATLAB. It has also shown some of their limitations and the 
benefit of improving the quality of the mfVEP signals. With the information provided, readers can begin practical implementation of the most common Prony methods, test the reliability of the results and, if applicable, research other methods more appropriate to their areas of research.

\section{Abbreviations}

AUC: Area under the ROC curve; DFT: Discrete Fourier transform; LS: Least squares; mfVEP: Multifocal visual-evoked potential; MPM: Matrix pencil method; MS: Multiple sclerosis; RMS: Root mean square; ROC: Receiveroperating-characteristic; SNR: Signal-to-noise ratio; SVD: Singular value decomposition; TLS: Total least squares

\section{Acknowledgements}

Thanks to Dr. Román Blanco (Universidad de Alcalá, Spain), for ceding the mfVEP recordings

\author{
Availability and requirements \\ Project name: MAT_PRONY \\ Project home page: Not applicable. \\ Operating system(s): Platform independent. \\ Programming language: Matlab. \\ Other requirements: Not applicable. \\ License: Matlab licences. \\ Any restrictions to use by non-academics: Not applicable.
}

\section{Funding}

Universidad de Alcalá grant "Diagnóstico precoz de neuropatías ópticas mediante análisis de registros de potenciales evocados visuales multifocales" (UAH GC2016-004) and Agencia Estatal de Investigación (AEI) - European Regional Development Fund (ERDF) grant "Investigación de la técnica de potenciales evocados visuales multifocales. Aplicación en estudios de evolución de esclerosis múltiple y evaluación de medicamentos" reference: DPI2017-88438-R (AEI/FEDER,UE). The funding organizations did not play any role in the design of the study, data collection and analysis, or preparation of the manuscript.

\section{Availability of data and materials}

The Matlab code is included in this published article.

\section{Authors' contributions}

AFR and LB conceived and designed the experiments; LdSR and JMRA performed the experiments; ELG and JMMJ revised the mathematics concepts; LB and AFR. wrote the paper; all the authors reviewed and approved the manuscript

\section{Ethics approval and consent to participate}

The mfVEP signals adquisition study protocol was approved by the Comité de Ética en Investigación Clínica del Hospital Universitario Príncipe de Asturias, Spain and adhered to the tenets of the Declaration of Helsinki. All participants provided written informed consent.

\section{Consent for publication}

Not applicable.

\section{Competing interests}

The authors declare that they have no competing interests.

\section{Publisher's Note}

Springer Nature remains neutral with regard to jurisdictional claims in published maps and institutional affiliations.
Received: 4 April 2018 Accepted: 7 November 2018

Published online: 26 November 2018

\section{References}

1. Prony R. Essai expérimental et analytique sur les lois de la dilatabilité des fluides élastiques, et sur celles de la force expansive de la vapeur de l'eau et de la vapeur de l'alkool, á différentes temperatures. J L'école Polytech. 1795; 1:24-76.

2. Householder, AS. On Prony's method of fitting exponential decay curves and multiple-hit survival curves. Oak Ridge National Laborator, 1950.

3. Markovsky I, Van Huffel S. Overview of total least-squares methods. Signal Process. 2007;87:2283-302. https://doi.org/10.1016/j.sigpro.2007.04.004.

4. Guillaume P, Schoukens J, Pintelon R. Sensitivity of roots to errors in the coefficient of polynomials obtained by frequency-domain estimation methods. IEEE Trans Instrum Meas. 1989;38:1050-6. https://doi.org/10.1109/ 19.46399.

5. Hua Y, Sarkar TK. Matrix pencil method for estimating parameters of exponentially damped/undamped sinusoids in noise. IEEE Trans Acoust. 1990;38:814-24. https://doi.org/10.1109/29.56027.

6. Lobos T, Leonowicz Z, Rezmer J, Schegner P. High-resolution spectrumestimation methods for signal analysis in power systems. IEEE Trans Instrum Meas. 2006:55:219-25. https://doi.org/10.1109/TIM.2005.862015.

7. Park SW, Schapery RA. Methods of interconversion between linear viscoelastic material functions. Part I-a numerical method based on Prony series. Int J Solids Struct. 1999;36:1653-75. https://doi.org/10.1016/S00207683(98)00055-9.

8. Sarkar T, Weiner D, Jain V. Some mathematical considerations in dealing with the inverse problem. IEEE Trans Antennas Propag. 1981;29:373-9. https://doi.org/10.1109/TAP.1981.1142573.

9. Fernández A, de Santiago L, Blanco R, Pérez-Rico C, Rodríguez-Ascariz JM, Barea R, Miguel-Jiménez JM, García-Luque JR, Ortiz del Castillo M, SánchezMorla EM, Boquete L. Filtering multifocal VEP signals using Prony's method. Comput Biol Med. 2015;56:13-9. https://doi.org/10.1016/j.compbiomed.2014. 10.023 .

10. Hansson M, Gänsler T, Salomonsson G. Estimation of single event-related potentials utilizing the Prony method. IEEE Trans Biomed Eng. 1996;43:97381. https://doi.org/10.1109/10.536898.

11. Chen SW. A two-stage discrimination of cardiac arrhythmias using a total least squares-based Prony modeling algorithm. IEEE Trans Biomed Eng. 2000;47:1317-27. https://doi.org/10.1109/10.871404.

12. Bhuiyan M, Malyarenko EV, Pantea MA, Seviaryn FM, Maev RG. Advantages and limitations of using matrix pencil method for the modal analysis of medical percussion signals. IEEE Trans Biomed Eng. 2013;60:417-26. https:// doi.org/10.1109/TBME.2012.2227318.

13. Bauman $\mathrm{G}$, Bieri $\mathrm{O}$. Matrix pencil decomposition of time-resolved proton MRI for robust and improved assessment of pulmonary ventilation and perfusion. Magn Reson Med. 2017;77:336-42. https://doi.org/10.1002/mrm. 26096.

14. Gopalakrishnan R, Machado AG, Burgess RC, Mosher JC. The use of contact heat evoked potential stimulator (CHEPS) in magnetoencephalography for pain research. J Neurosci Methods. 2013;220:55-63. https://doi.org/10.1016/j. jneumeth.2013.08.015.

15. Goodman D. Brian: a simulator for spiking neural networks in Python. Front Neuroinform. 2008;2:5. https://doi.org/10.3389/neuro.11.005.2008.

16. Meyer R, Obermayer K. Pypet: a Python toolkit for data management of parameter explorations. Front. Neuroinform. 2016;10. https://doi.org/10. 3389/fninf.2016.00038

17. Sen SK, Shaykhian GA. MatLab tutorial for scientific and engineering computations. Nonlinear Anal Theory, Methods Appl. 2009:e1005-20. https://doi.org/10.1016/j.na.2009.01.069.

18. Ihlen EAF. Introduction to multifractal detrended fluctuation analysis in Matlab. Front Physiol. 2012;3. https://doi.org/10.3389/fphys.2012.00141.

19. Miguel-Jiménez JM, Boquete L, Ortega S, Cordero CA, Barea R, Blanco R. mfERG_LAB: software for processing multifocal electroretinography signals. Comput Methods Prog Biomed. 2012;108:377-87. https://doi.org/10.1016/j. cmpb.2012.02.013.

20. Perakakis P, Joffily M, Taylor M, Guerra P, Vila J. KARDIA: a Matlab software for the analysis of cardiac interbeat intervals. Comput Methods Prog Biomed. 2010;98:83-9. https://doi.org/10.1016/j.cmpb.2009.10.002.

21. Saber E. An introduction to difference equations. New York: Springer-Verlag; 2005. https://doi.org/10.1007/0-387-27602-5. 
22. Reddy DC. Biomedical signal processing: principles and techniques. New York: McGraw-Hill; 2005.

23. Van Huffer S, Zha H. The total least squares problem. Handb. Stat. 1993;9: 377-408.

24. Van Huffel S, Vandewalle J. The total least squars problem: computational aspects and analysis. Philadelphia: Siam; 1991.

25. Yang WY, Cao W, Chung TS, Morris J. Applied numerical methods using MATLAB. Hoboken: Wiley; 2005.

26. Gantmacher FR. The theory of matrices, vol. 2. Moscow: GITTL; 1953.

27. Sarkar TK, Pereira O. Using the matrix pencil method to estimate the parameters of a sum of complex exponentials. IEEE Antennas Propag Mag. 1995;37:48-55. https://doi.org/10.1109/74.370583.

28. Baseler HA, Sutter EE, Klein SAA, Carney T. The topography of visual evoked response properties across the visual field. Electroencephalogr Clin Neurophysiol. 1994;90:65-81. https://doi.org/10.1016/0013-4694(94)90114-7.

29. Müller PL, Meigen T. M-sequences in ophthalmic electrophysiology. J Vis. 2016;16(1):15

30. Mitrofanov G, Priimenko V. Prony filtering of seismic data. Acta Geophys. 2015;63:652-78. https://doi.org/10.1515/acgeo-2015-0012.

31. Lander $P$, Jones $D$, Berbari $E$, Lazzara R. Time-frequency structure of the high-resolution ECG. J Electrocardiol. 1994;27:207-12. https://doi.org/10. 1016/S0022-0736(94)80093-6.

32. Demiralp T, Ademoglu A, Istefanopulos Y, Gülçür HO. Analysis of eventrelated potentials (ERP) by damped sinusoids. Biol Cybern. 1998;78:487-93. https://doi.org/10.1007/s004220050

33. Slivinskas V, Šimonyte V. On the foundation of Prony's method. In: Stoch. Control, Elsevier; 1987. p. 121-6. https://doi.org/10.1016/B978-0-08-033452-3. 50025-9.

34. James Hu SL, Yang WL, Li HJ. Signal decomposition and reconstruction using complex exponential models. Mech Syst Signal Process. 2013;40:42138. https://doi.org/10.1016/j.ymssp.2013.06.037.

35. Davis TA, Duff IS. An unsymmetric-pattern multifrontal method for sparse LU factorization. SIAM J Matrix Anal Appl. 1997;18:140-58. https://doi.org/10 1137/S0895479894246905.

36. Demmel JW. Applied numerical linear algebra, SIAM; 1997. https://doi.org/ 10.1137/1.9781611971446.

37. Katsikis VN, Pappas D, Petralias A. An improved method for the computation of the Moore-Penrose inverse matrix. Appl Math Comput. 2011;217:9828-34. https://doi.org/10.1016/j.amc.2011.04.080

38. Ataei A. Improved Qrginv algorithm for computing Moore-Penrose inverse matrices. ISRN Appl Math. 2014;2014:1-5. https://doi.org/10.1155/2014/641706.

39. Petković MD, Stanimirović PS. Two improvements of the iterative method for computing Moore-Penrose inverse based on Penrose equations. J Comput Appl Math. 2014;267:61-71. https:/doi.org/10.1016/j.cam.2014.01.034.

40. Fierro RD, Golub GH, Hansen PC, O'Leary DP. Regularization by truncated total least squares. SIAM J Sci Comput. 1997;18:1223-41. https://doi.org/10. 1137/S1064827594263837.

41. Markovsky I. Bibliography on total least squares and related methods. Stat Interface. 2010;3:329-34.

42. Lampe J, Voss H. Large-scale Tikhonov regularization of total least squares. J Comput Appl Math. 2013;238:95-108. https://doi.org/10.1016/j. cam.2012.08.023.

43. Xie, P., Wei, Y., Xiang, H. Perturbation analysis and randomized algorithms for large-scale total least squares problems. arXiv preprint arXiv:1401.6832, 2014

44. Pan VY. Solving a polynomial equation: some history and recent progress. SIAM Rev. 1997;39:187-220. https://doi.org/10.1137/S0036144595288554.

45. Hansen E, Patrick M, Rusnak J. Some modifications of Laguerre's method. BIT. 1997;17:409-17. https://doi.org/10.1007/BF01933450.

46. Madsen K. A root-finding algorithm based on Newton's method. BIT. 1973; 13:71-5. https://doi.org/10.1007/BF01933524.

47. Golub GH, van der Vorst HA. Eigenvalue computation in the 20th century. J Comput Appl Math. 2000;123:35-65.

48. Chandrasekaran S, Gu M, Xia J, Zhu JA. Fast QR algorithm for companion matrices. In: Recent Adv. Matrix Oper. Theory, Birkhäuser Basel, Basel; 2007. p. 111-43. https://doi.org/10.1007/978-3-7643-8539-2_7.

49. Saad Y. Numerical methods for large eigenvalue problems, Society for Industrial and Applied Mathematics; 2011. https://doi.org/10.1137/1. 9781611970739
50. Schmidt R. Multiple emitter location and signal parameter estimation. IEEE Trans Antennas Propag. 1986;34:276-80. https://doi.org/10.1109/TAP.1986. 1143830.

51. Roy R, Paulraj A, Kailath T. ESPRIT—A subspace rotation approach to estimation of parameters of cisoids in noise. IEEE Trans Acoust Speech Signal Process. 1986;34:1340-2. https://doi.org/10.1109/TASSP.1986.1164935.

52. Potts $\mathrm{D}$, Tasche M. Fast ESPRIT algorithms based on partial singular value decompositions. Appl Numer Math. 2015;88:31-45. https://doi.org/10.1016/j. apnum.2014.10.003.

53. Potts $\mathrm{D}$, Tasche M. Parameter estimation for nonincreasing exponential sums by Prony-like methods. Linear Algebra Appl. 2013;439:1024-39. https://doi.org/10.1016/j.laa.2012.10.036.

54. Nigmatullin RR, Osokin SI, Toboev VA. NAFASS: discrete spectroscopy of random signals. Chaos, Solitons Fractals. 2011;44:226-40. https://doi.org/10. 1016/i.chaos.2011.02.003.

55. Nigmatullin RR, Khamzin AA, Machado JT. Detection of quasi-periodic processes in complex systems: how do we quantitatively describe their properties? Phys Scr. 2013;89:015201. https://doi.org/10.1088/0031-8949/89/ 01/015201.

56. Nigmatullin RR, Gubaidullin IA. NAFASS: fluctuation spectroscopy and the Prony spectrum for description of multi-frequency signals in complex systems. Commun Nonlinear Sci Numer Simulat. 2018:56:252-69. https://doi. org/10.1016/j.cnsns.2017.08.009.

57. Kumaresan R, Feng Y. FIR prefiltering improves Prony's method. IEEE Trans Signal Process. 1991;39:736-41. https://doi.org/10.1109/78.80860.

58. Azarov E Vashkevich M, Petrovsky A. Instantaneous harmonic representation of speech using multicomponent sinusoidal excitation. Proc Annu Conf Int Speech Commun Assoc Interspeech. 2013:1697-701.

59. Rezaiesarlak R, Manteghi M. Short-time matrix pencil method for chipless RFID detection applications. IEEE Trans Antennas Propag. 2013;61:2801-6. https://doi.org/10.1109/TAP.2013.2238497.

60. Garoosi V, Jansen BH. Development and evaluation of the piecewise Prony method for evoked potential analysis. IEEE Trans Biomed Eng. 2000;47:154954. https://doi.org/10.1109/10.887935.
Ready to submit your research? Choose BMC and benefit from:

- fast, convenient online submission

- thorough peer review by experienced researchers in your field

- rapid publication on acceptance

- support for research data, including large and complex data types

- gold Open Access which fosters wider collaboration and increased citations

- maximum visibility for your research: over $100 \mathrm{M}$ website views per year

At BMC, research is always in progress.

Learn more biomedcentral.com/submissions 